\title{
Combination of DFT and Multitone Modulation in OFDM Systems
}

\author{
G. Cariolaro \\ Dipartimento di Elettronica ed Informatica, Universitá di Padova \\ Via Gradenigo 6/A, 35131 Padova, Italy
}

\section{A. Costanzi \\ Italtel S.p.A, Via de Tocqueville, 1320154 Milano, Italy}

In the implementation of OFDM systems one of the most consolidated schemes consists of an $N$-point DFT processor and an $N$-input $N$-output polyphase network, where $N$ is the number of channels. Another recently developed scheme, which is attractive for its conceptual simplicity, consists of a polyphase filter, a multitone modulator (MTM) with carrier $\exp \left(2 \pi\left(F_{0} t\right)^{2} / 2 N\right)$, and an equalizer.

In this paper we derive a combination of the two previous schemes, where the number of channels $\mathrm{N}$ is factored in the form $N=M \cdot I$. The composite scheme consists of: 1) an I-point DFT processor; 2) a bank of $I$ different MTM and 3) a bank of $I$ different polyphase filters; and 4) a bank of $I$ identical MTM with carriers.

The composite scheme degenerates into the previous schemes in the extreme cases provided by factorization $M \cdot I$. Specifically, the DFT scheme is obtained with $M=1$ and $I=N$, and the MTM scheme with $\mathrm{M}=\mathrm{N}$ and $\mathrm{I}=1$. Here the attention is for the intermediate cases of factorization.

\section{Abbreviations and symbolism}

DFT:

MTM:

SCS:

$\mathrm{S} / \mathrm{P}$ :

$\mathrm{P} / \mathrm{S}$ :

$N$ :

$M \cdot I=N$ :

$F:$

$F_{0}=N F:$

$F_{1}=M F$ :

$\gamma(r)=W_{2 r^{2}}^{r^{2}}$ :

$\lambda(r)=W_{2 M}^{-r^{2}}:$

$\varphi_{i}(r)=W_{N}^{i \beta_{M}^{(r)}}:$

$\beta_{M}(r)$ :
Discrete Fourier Transform

MultiTone Modulation

Standard Complex Scheme

serial-to-parallel conversion parallel-to-serial conversion number of complex channels ( $2 N$ real channels) factorization of $N$ "low rate" (rate of individual channels) "high rate" (rate of multiplexed channels) "medium rate"

MTM carrier

MTM carrier

MTM carrier

rest of the integer division of $r$ by $M$ 


$$
\mathbb{Z}:
$$$$
Z(T)=|n T| n \in Z\}
$$

set of integers

\section{Introduction}

In recent years several schemes for OFDM systems have been proposed with the goal of reducing the hardware involved.

In principle, an OFDM scheme is similar to a transmultiplexer, i.e. the digital translation of a time division multiplex (TDM) signal into a frequency-division multiplex (FDM) signal of $N$ voice channels, which received a considerable interest at the beginning of the last decade. Transmultiplexer techniques are well documented by a Special Issue of the IEEE Trans. On Communications [1] and by an IEEE Proceedings survey [2].

For both transmultiplexer and OFDM systems one of the most consolidated schemes [3] [7] consists of an $N$-point DFT processor and an $N$-input $N$-output polyphase network, both working at the "low rate" $F$ of the individual channels (Fig. la).

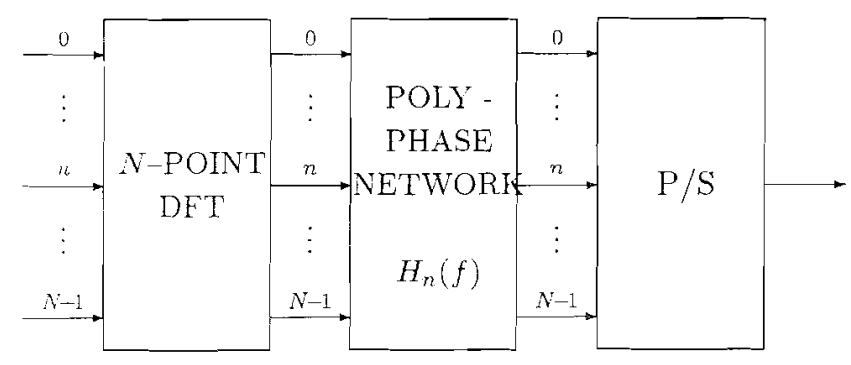

a)

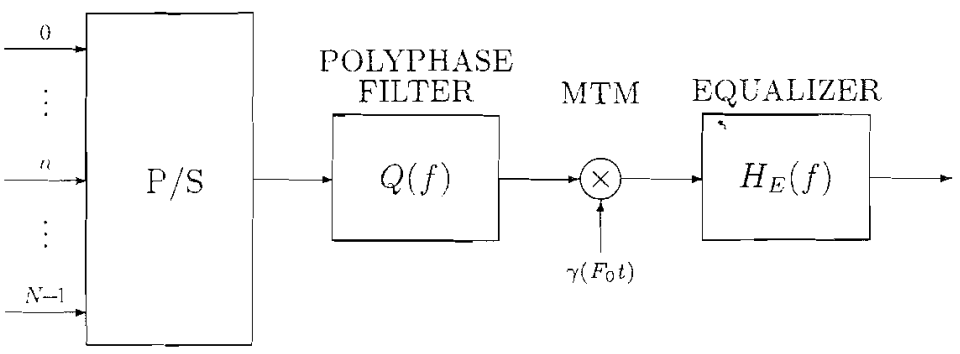

b)

Figure 1 - The OFDM scheme: a) via DFT and polyphase network, and b) via polyphase filter and MTM. 
Another recently developed scheme [8] [9], which is attractive for its conceptual simplicity, consists of (Fig. 1b) a polyphase filter, a modulator with carrier $\exp \left(j 2 \pi\left(F_{0} t\right)^{2} / 2 N\right)$, and an equalizer, all working at the "high rate" $F_{0}=N F$, where N is the number of channels and $\mathrm{F}$ is the rate of the individual channel. The polyphase filter is all-pass with constant phase in each sideband of the resulting OFDM signal and the modulator produces $\mathrm{N}$ simultaneous frequency-shifts, thus providing a multitone modulation (MTM) of the incoming signal.

In this paper we derive a combination of the two aforementioned schemes, where the number of channels $N$ is factored in the form

$$
N=M \cdot I
$$

with $M$ and $I$ positive integers. The composite scheme consists of (Fig. 2):

1) an $I$-point DFT processor;

2) a bank of $I$ different MTM with carriers

$$
\varphi_{i}\left(F_{1} t\right)=W_{N}^{\mathrm{P}_{M}\left(F_{1} t\right)}, 0 \leq i \leq I-1
$$

where $\beta_{M}(n)$ denotes the remainder of the integer division of $n$ by $M$;

3) a bank of $I$ different polyphase filters;

4) a bank of $I$ identical MTM with carriers

$$
\gamma\left(F_{1} t\right)=W_{2 M}^{\left(F_{1} t\right)^{2}}
$$

All the components 1) to 4 ) work at the "medium rate" $F_{1}=M F=F_{0} / I$.

1 The syntheses presented in Figs. 1 and 2 are referred to the OFDM Standard Complex Scheme; N represents the number of complex data channels (see Section III). 


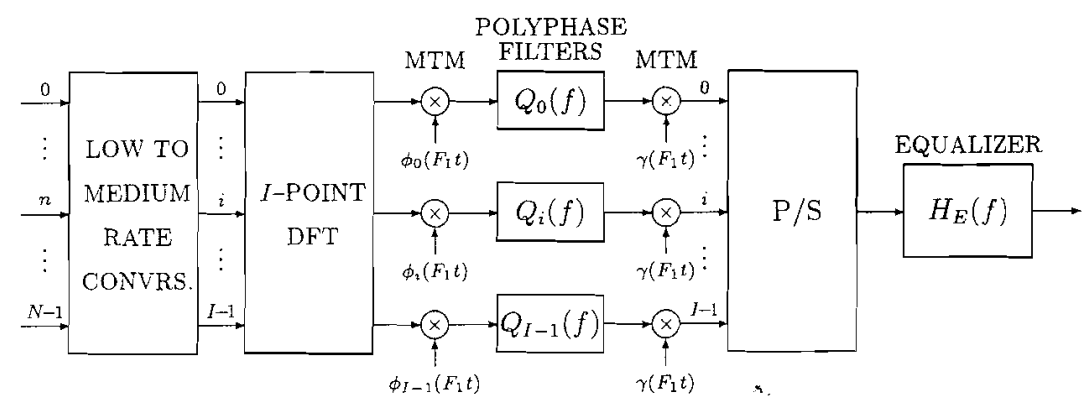

Figure 2 - The "composite" OFDM scheme with both DFT and MTM.

The composite scheme of Fig. 2 degenerates into the previous schemes in the extreme cases provided by factorization (1). Specifically, the DFT scheme is obtained with $M=1$ and $I=N$, and the MTM scheme with $M=N$ and $I=1$. Here the attention is for the intermediate cases of factorization. To be specific we consider one of the most attractive application of the OFDM technique, the digital television, in which the number of channels may run from $N=128$ to $N=1024$ and recently also $N=2048$ and $N=8196$ [11]. Some cases the possible factorizations, with $N, I$ and $M$ powers of 2 , are listed in Tab. 1. We note in particular that when $I=4$ or $I=8$, the $I$-point DFT processor has a negligible computational complexity and hence the attention for the complexity reduction may be concentrated on the rest of the scheme.

\begin{tabular}{rr|rr|rr|rr}
\hline \multicolumn{2}{c|}{$N=128$} & \multicolumn{2}{c|}{$N=256$} & \multicolumn{2}{c|}{$N=512$} & \multicolumn{2}{c}{$N=1024$} \\
\hline $\mathrm{M}$ & $\mathrm{I}$ & $\mathrm{M}$ & $\mathrm{I}$ & $\mathrm{M}$ & $\mathrm{I}$ & $\mathrm{M}$ & $\mathrm{I}$ \\
\hline 128 & 1 & 256 & 1 & 512 & 1 & 1024 & 1 \\
\hline 64 & 2 & 128 & 2 & 256 & 2 & 512 & 2 \\
\hline 32 & 4 & 64 & 4 & 128 & 4 & 256 & 4 \\
\hline 16 & 8 & 32 & 8 & 64 & 8 & 128 & 8 \\
\hline 8 & 16 & 16 & 16 & 32 & 16 & 64 & 16 \\
\hline 4 & 32 & 8 & 32 & 16 & 32 & 32 & 32 \\
\hline 2 & 64 & 4 & 64 & 8 & 64 & 16 & 64 \\
\hline 1 & 128 & 2 & 128 & 4 & 128 & 8 & 128 \\
\hline & & 1 & 256 & 2 & 256 & 4 & 256 \\
\hline & & & & 1 & 512 & 2 & 512 \\
\hline & & & & & & 1 & 1024 \\
\hline
\end{tabular}

Table 1 - Possible decompositions $N=M . I$ for some values of $N$. 
This paper is organized as follows. Section II deals with preliminaries concerning signal and system representation. In Sections III and IV we derive the composite scheme following the time-domain approach, which is less standard, but more straightforward than the zeta-domain approach. ${ }^{2}$ In Sections V and VI we discuss the components of the composite scheme and examine their complexity, taking as reference the complexity of the scheme via DFT.

All the developments are based on complex signals and complex processors. The resulting complex scheme of Fig. 2 can be easily implemented via real operations .

\section{Preliminaries on Signals and Systems}

In this section we introduce some preliminaries on the time-domain representation of signals and systems, which will be useful in the following.

\section{A Discrete-time signals}

A discrete-time signal will be denoted in the forms

$$
x(t), t \in Z\left(T_{0}\right) \text { or } x\left(n T_{0}\right), n \in \mathbb{Z},
$$

where $Z\left(T_{0}\right)=\left\{\ldots,-T_{0}, 0, T_{0}, 2 T_{0}, \ldots\right\}$ is the time-domain, $T_{0}$ is the time-spacing between two consecutive values, and its reciprocal $F_{0}=1 / T_{0}$ represents the rate expressed in number of values per second. ${ }^{3}$

The zeta-transform of (4) is defined by

$$
X(z)=\sum_{n=-\infty}^{+\infty} T_{0} x\left(n T_{0}\right) z^{-n}
$$

from which one gets the Fourier transform by setting $z=\exp \left(j 2 \pi f T_{0}\right)$, namely ${ }^{4}$

2 Compare the two approaches in [7] and [8] for the deduction of the scheme via MTM.

3 For the presence of rate alterations in a OFDM system, the standard normalization $T_{0}=F_{0}+1$ is not convenient.

4 Hereafter the tilde will be dropped and the Fourier transform will be denoted by the same symbol $X(f)$ used for the zeta-transform. 


$$
\tilde{X}(f)=X\left(e^{\left.j 2 \pi f T_{0}\right)}=\sum_{n=-\infty}^{+\infty} T_{0} x\left(n T_{0}\right) e^{j 2 \pi f n T_{0}}\right.
$$

Expression (5b) has a period equal to the signal rate $F_{0}$.

When the signal $x\left(n T_{0}\right)$ is periodic with period, say $N T_{0}$, in place of $(5 \mathrm{~b})$ we can consider the discrete Fourier transform (DFT), namely

$$
X(q)=\frac{1}{N} \sum_{n=0}^{N-1} x\left(n T_{0}\right) W_{N}^{-q n}, W_{N} \triangleq e^{j 2 \pi / N}
$$

whose inverse is

$$
x\left(n T_{0}\right)=\sum_{q=0}^{N-1} X(q) W_{N}^{q n}
$$

Remarkable examples of periodic discrete-time signals are provided by the MTM carriers (2) and (3).

1

\section{B Single-tone and multitone modulations}

A linear modulation for discrete time signals can be defined by the relationship:

$$
y(t)=c(t) x(t), \quad t \in Z\left(T_{0}\right),
$$

where $x(t)$ is the modulating signal, $y(t)$ the modulated signal, and the carrier $c(t)$ is periodic (of period $N T_{0}$ ). In the frequency and zeta domain (6) becomes, respectively $[8]$ :

$$
Y(f)=\sum_{q=0}^{N-1} C(q) X(f-q F) \quad, \quad F=F_{0} / N
$$




$$
Y(z)=\sum_{q=0}^{N-1} C(q) X\left(z W_{N}^{-q}\right)
$$

where $C(q)$ is the DFT of the carrier.

For complex signals the canonical modulation is provided by the exponential

$$
c\left(n T_{0}\right)=e^{j 2 \pi q_{l 0} F_{n} T_{0}}=W_{N}^{q_{0} n}, N=\frac{1}{F T_{0}}
$$

which causes a single frequency shift of $q_{0} F$ for the incoming signals (single tone modulation); in fact, in this case $C(q)=1$ for $q=q_{0}$ and $C(q)=0$ for $q \neq q_{0}$. The opposite case happens when the DFT $C(q) \neq 0$ for any q so that the modulation causes $\mathrm{N}$ simultaneously frequency shifts multiple of $\mathrm{F}=\mathrm{F}_{0} / N$ (multitone modulation). Examples of MTM are provided by the carriers (2) and (3) (see Section VI).

\section{Filters and interpolating filters}

Let $Z\left(T_{0}\right)$ and $Z(T)$ be two discrete domains with $T=N T_{0}$ and let $\mathrm{t}$ and $\mathrm{t}_{0}$ denote the running time in $Z(T)$ and $Z\left(T_{0}\right)$, respectively. Then, a filter with input $x(t)$ on $Z(T)$ and output $y\left(t_{0}\right)$ on $Z\left(T_{0}\right)$ can be defined by the relationship

$$
y\left(t_{0}\right)=\sum_{r \in Z(T)} \operatorname{Tg}\left(t_{0}-\tau\right) x(\tau), \quad t_{0} \in Z\left(T_{0}\right)
$$

where $g\left(t_{0}\right), t_{0} \in Z\left(T_{0}\right)$, is the kernel characterizing the filter.

In particular for $N=1$, i.e. $T=T_{0}$, we get an ordinary filter and for $N>1$ an interpolating filter, which provides a signal-rate increase from $F=1 / T$ into $F_{0}=N F$. In any case the kernel $g\left(t_{0}\right)$ is defined on the output time domain $Z\left(T_{0}\right)$.

\section{D $S / P$ and $P / S$ conversions}

An arbitrary discrete-time signal $x\left(t_{0}\right), t_{0} \in Z\left(T_{0}\right)$, can be decomposed into $N$ lower rate discretetime signals $x_{n}(t), t \in Z(T)$, with $T=N T_{0}$, by the serial-to-parallel (S/P) conversion, which is defined by 


$$
x_{n}(t)=x\left(t+n T_{0}\right), \quad t \in Z(T), 0 \leq n \leq N-1 .
$$

Conversely, $N$ discrete-time signals $x_{n}(t), t \in Z(T)$, can be combined into a single higher rate discrete-time signal $\mathrm{x}\left(\mathrm{t}_{0}\right), t_{0} \in Z\left(T_{0}\right)$, by the parallel-to-serial $(\mathrm{P} / \mathrm{S})$ conversion, which is defined by

$$
x\left(t+n T_{0}\right)=x_{n}(t), t \in Z(T), 0 \leq n \leq N-1
$$

In the zeta-domain the above relationships become respectively [8]

$$
\begin{gathered}
X_{n}\left(z^{N}\right)=\frac{1}{N} \sum_{k=0}^{N-1} X\left(z W_{N}^{-k}\right) z^{n} W_{N}^{-k n} \\
X(z)=\sum_{n=0}^{N-1} X_{n}\left(z^{N}\right) z^{-n}
\end{gathered}
$$

Essentially, a $\mathbf{P} / \mathrm{S}$ conversion is a time division multiplexing and an $\mathrm{S} / \mathrm{P}$ conversion is a timedivision demultiplexing.

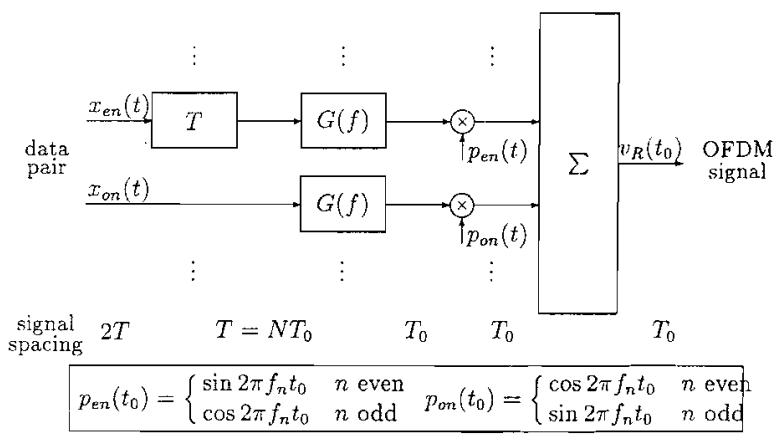

Figure 3 -Basic OFDM transmitter scheme for $N$ data pairs with symbol period $2 T$. 


\section{The OFDM System}

An OFDM transmitter conveys $N$ data complex symbol sequences belonging to a same constellation, e.g. with a QAM format, into a single higher rate sequence according to the scheme of Fig. 3, where $2 T$ denotes the symbol period. For convenience the $N$ complex sequences are decomposed into real and imaginary parts, thus obtaining $N$ pairs of real data sequences $x_{e n}(t), x_{o n}(t)$. The even data $x_{e n}(t)$ are first delayed by $T$ and then interpolated by a filter $G(f)$; the odd data are directly interpolated by $G(f)$. After the interpolation to the rate $F_{0}=N F$, where $\mathrm{F}=1 / \mathrm{T}$, the data are modulated by the carriers $\sin 2 \pi f_{n} t_{0}$ or $\cos 2 \pi f_{n} t_{0}$ according the alternate rule listed in Fig. 3 . The frequencies fn are equally spaced, specifically

$$
f_{n}=f_{0}+n \frac{1}{2} F, f_{0}=\frac{1}{4} F
$$

In the ideal case the frequency responses of the interpolating filters $G(f)$ are given by the square root of a raised-cosine characteristic with Nyquist frequency $\frac{1}{4} F$.

For purpose of syntheses, it is convenient to modify the basic scheme of Fig. 3 in four parts [7] (Fig. 4):

1) data pairing (DP)

2) premodulations and ordering $(\mathrm{P} \& \mathrm{O})$,

3) standard complex scheme (SCS).

4) real part $\mathfrak{K}[\cdot]$.

The DP is essentially a $\mathrm{P} / \mathrm{S}$ of the data pairs into a single complex signal, according to

$$
x_{n}(t)=\left\{\begin{array}{cl}
-j x_{o n}(t)+x_{e n}(t-T) & n \text { even } \\
x_{o n}(t)-j x_{e n}(t-T) & n \text { odd }
\end{array}\right.
$$

The $\mathrm{P} \& \mathrm{O}$ consists of

a) a first modulation given by

$$
c_{n}(k T)=x_{n}(k T)(j)^{k}
$$




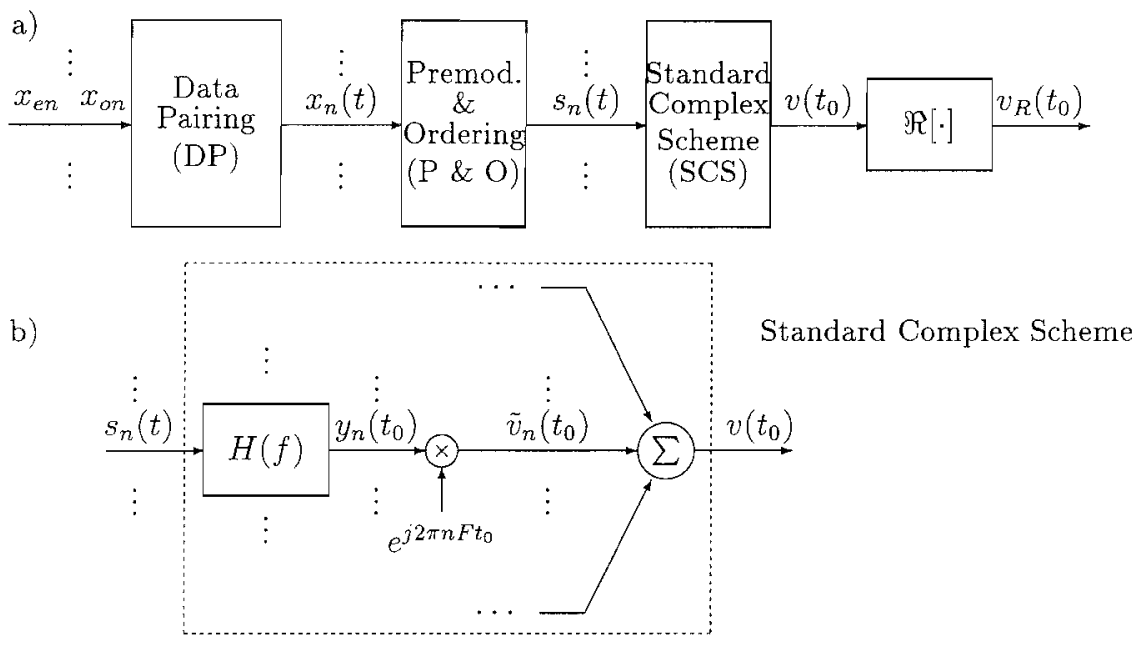

Figure 4 -Subdivision of the basic OFDM scheme, and b) the Standard Complex Scheme (SCS).

b) an ordering given by

$$
d_{n}(t)= \begin{cases}c * 2 n(t) & n=0,1, \ldots, \frac{1}{2} N-1 \\ c_{N-2 n-1}(t) & n=0,1, \ldots, \frac{1}{2} N-1\end{cases}
$$

c) a second modulation given by

$$
s_{n}(k T)= \begin{cases}d_{n}(k T) & n=0,1, \ldots, \frac{1}{2} N-1 \\ d_{n}(k T)(-1)^{k} & n=\frac{1}{2} N, \ldots, N-1\end{cases}
$$

The SCS converts the $N$ complex signals $s_{n}(t), t \in Z(T)$, in a complex OFDM signal $v\left(t_{0}\right)$, whose real part $v_{R}\left(t_{0}\right)$ represents the final signal to be transmitted. Its operations are

1) $N$ identical interpolations from the time-spacing $T$ to $T_{0}$ by a complex filter $H(f)$, for all the $s_{n}(t)$;

54 
2) $N$ single tone modulations with carriers $\exp \left(j 2 \pi n F t_{0}\right)=W_{N}^{n}, \mathrm{n}=0,1, \ldots, \mathrm{N}-1$.

3) a final summation.

The complex filter $H(f)$ is an offset version of the original filter $G(f)$, namely

$$
H(f)=G\left(f-\frac{1}{4} F\right)
$$

Referring to a square-root raised cosine characteristic the bandwidth of $G(f)$ is (Fig. 5)

$$
B_{G}=\left(-(1+\alpha) \frac{1}{4} F,(1+\alpha) \frac{1}{4} F\right)
$$

where $\alpha$ is the rolloff factor. For the offset version $H(f)$ the bandwidth becomes 5

$$
\mathcal{B}_{0}=\mathcal{B}_{G}+\frac{1}{4} F=\left(-\alpha \frac{1}{4} F,(2+\alpha) \frac{1}{4} F\right)
$$

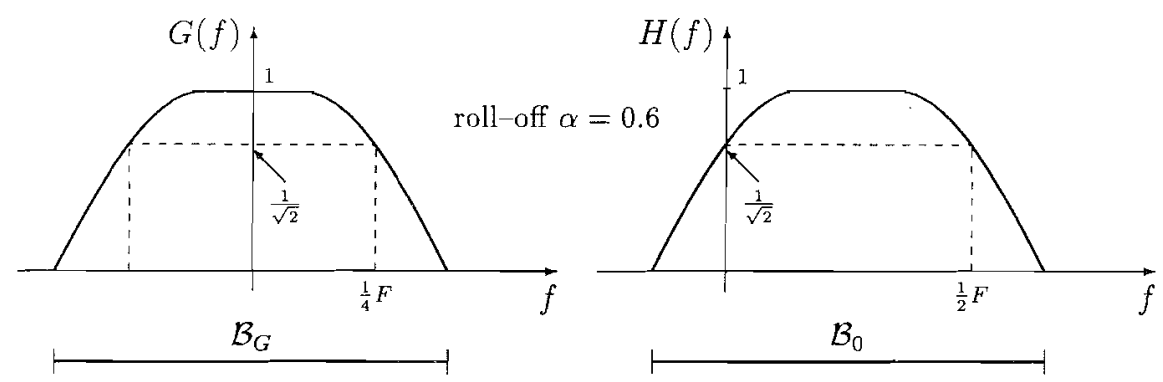

Figure 5 - The original square root raised cosine frequency characteristic $G(f)$ and its shifted version $H(f)$.

We remark that in DP and $\mathrm{P} \& O$ the computational complexity is irrelevant, hence the attention for the synthesis will be confined to the SCS.

$5 B_{G}+1 / 4 F$ denotes the interval $B_{G}$ shifted by $1 / 4 F$. 


\section{A Equations of the SCS}

The operations in the SCS are governed by the following time domain equations:

1) interpolation with filter $H(f)$ :

$$
y_{n}\left(r T_{0}\right)=\sum_{k=-\infty}^{+\infty} T h\left(r T_{0}-k T\right) s_{n}(k T),
$$

where $h\left(k T_{o}\right)$ is the impulse response of the filter;

2) single-tone modulations with frequency-shifts $n F$ :

$$
\tilde{\boldsymbol{v}}_{n}\left(r T_{0}\right)=y_{n}\left(r T_{0}\right) W_{N}^{r n}
$$

3) summation, which yields the complex OFDM signal

$$
v\left(r T_{0}\right)=\sum_{n=0}^{N-1} \widetilde{v}_{n}\left(r T_{0}\right)
$$

Combining 1), 2) and 3) we find the following relationship, linking the complex OFDM signal to the input signals $s_{n}(t)$ :

$$
v\left(r T_{0}\right)=\sum_{n=0}^{N-1} \sum_{k=-\infty}^{+\infty} T h\left(r T_{0}-k T\right) W_{N}^{n} S_{n}(k T) .
$$

\section{Derivation of the Composite Scheme}

The derivation technique is based on a suitable rearrangement of the time-domain equations describing the OFDM-SCS obtained in the previous section, in particular the overall relationship (19). We decompose the number of channels to be multiplexed in the form

$$
N=M . I
$$


Correspondingly, we introduce the following signal rates and time-spacings

"low rate" $F$

$$
\begin{aligned}
& \mathrm{T}=\mathrm{l} / \mathrm{F} \\
& \mathrm{T}_{l}=l / F_{l} \\
& \mathrm{~T}_{0}=l / F_{0}
\end{aligned}
$$

"medium rate" $F_{l}=M F$

"high rate" $F_{0}=N F=I F_{1}$

(20a)

where $F$ is the rate of the individual channels, $F_{0}$ the rate of the multiplexed signal, and $F_{1}$ the operating rate of components in the composite scheme. The time spacings are related as follows:

$$
T=N T_{0}=M T_{1}, \quad T_{1}=I T_{0}
$$

\section{A Possibility of output equalization}

It is fundamental to remark that the $N$ identical interpolating filters $H(f)$ in the scheme of Fig. 4 can be replaced by arbitrary, possibly different, interpolating filters $G_{n}(f)$ with band $\mathcal{B}_{0}$, provided that the complex OFDM signal $y\left(r T_{0}\right)$ be correspondingly equalized

(Fig. 6). The equalization condition is

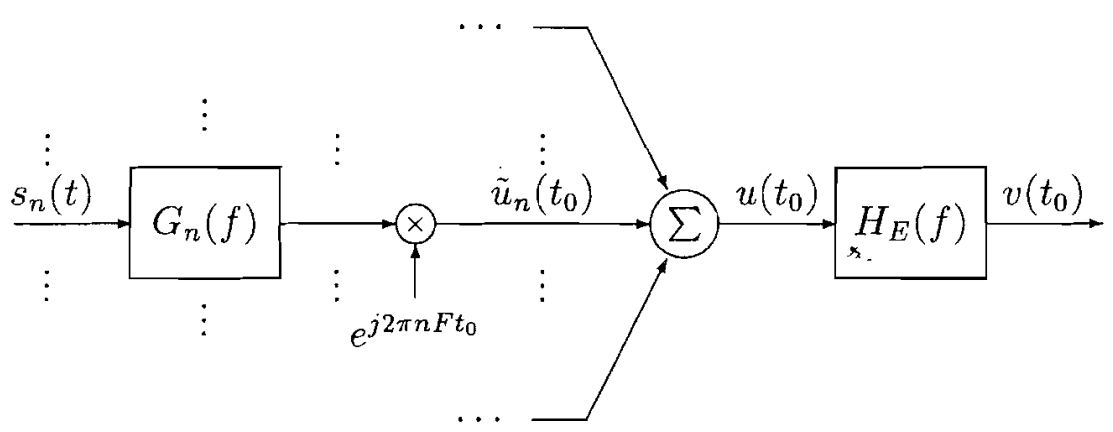

Figure 6 - The modified Standard Complex Scheme with modified filters and a final equalizer. 
given by

$$
H_{E}(f)= \begin{cases}\frac{H(f-n F)}{G_{n}(f-n F)}, & f \in B_{n} n=0,1, \ldots, N-1 \\ \text { arbitrary } & , \text { otherwise }\end{cases}
$$

where

$$
\mathscr{B}_{n}=B_{0}+n F
$$

are the bands after the frequency shifts $n F$.

The unequalized complex OFDM signal is given by

$$
u\left(r T_{0}\right)=\sum_{n=0}^{N-1} \sum_{-\infty}^{+\infty} T g_{n}\left(r T_{0}-k T\right) W_{N}^{n} s_{n}(k T)
$$

The complex OFDM signal is then obtained by the equalization relationship

$$
v\left(r T_{0}\right)=h_{E} * u\left(r T_{0}\right)
$$

Equation (22) is the starting point for the derivation of the composite scheme. First we assume that the filters $g_{n}\left(k T_{0}\right)$ are arbitrary and then we complete their definition as the derivation proceeds.

\section{B Introduction of medium-rate signals}

Let $N=M I$. The first modification of (22) is made by grouping the $N$ low-rate signals $s_{n}(t), t \in Z(T)$, into I medium rate signals (Fig. 7):

$$
a_{0}(t), a_{1}(t), \ldots, a_{I-1}(t), \quad t \in Z(T), \quad T_{1}=I T .
$$




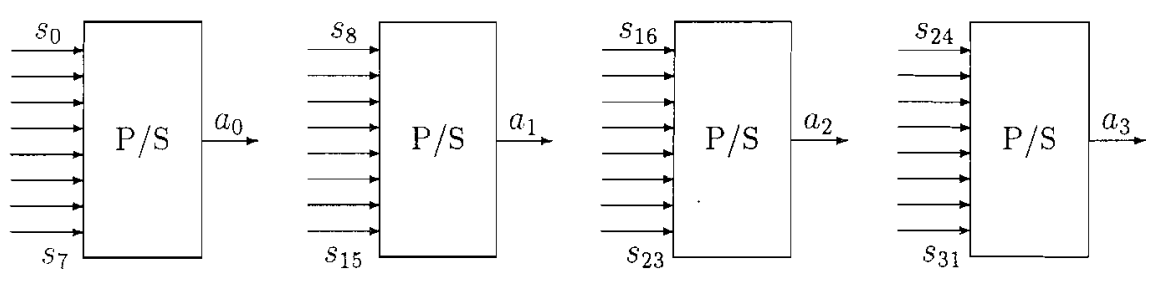

Figure 7 -The conversion of $N$ low-rate signals $s_{n}$ into / medium-rate signals $a_{i}$ for $N=32, M=8, I=4$.

This is achieved by writing the index $n$ in the form

$$
n=i M+m, \quad 0 \leq i \leq I-1, \quad 0 \leq m \leq M-1,
$$

namely

$$
a_{i}\left(t+m T_{1}\right)=s_{i M+m}(t), t \in Z(T),
$$

where the new argument $t+m T_{1}$ with $t \in Z(T)$ and $m \in\{0,1, \ldots, M-1\}$ runs on the medium rate set $Z\left(T_{1}\right)$. The $i$-th signal is obtained by the $\mathrm{P} / \mathrm{S}$ conversion of the $M$ consecutive low rate signals $s_{i M+m}(t), 0 \leq m \leq M-1$. With the previous replacements, the unequalized complex OFDM signal becomes:

$$
\begin{aligned}
u\left(r T_{0}\right) & =\sum_{k=-\infty}^{+\infty} \sum_{i=0}^{I-1} \sum_{m=0}^{M-1} T g_{i M+m}\left(r T_{0}-k T\right) W_{N}^{(i M+m)} s_{i M+m}(k T) \\
& =\sum_{k=-\infty} \sum_{i=0}^{I-1} \sum_{m=0}^{M-1} T g_{i M+m}\left(r T_{0}-k T\right) W_{N}^{r i} W_{N}^{m} a_{i}\left(k T+m T_{1}\right)
\end{aligned}
$$

\section{Introduction of the DFT}

Hereafter we assume that the filters $g_{n}\left(t_{0}\right)$ satisfy the condition 


$$
g_{i M+m}\left(t_{0}\right)=g_{m}\left(t_{0}\right) \quad \text { independent of } i
$$

Hence, (26) can be written in the form

$$
u\left(r T_{0}\right)=\sum_{k=-\infty}^{+\infty} \sum_{m=0}^{M-1} \operatorname{Tg}_{m}\left(r T_{0}-k T\right) W_{N}^{m} A_{r}\left(k T+m T_{1}\right)
$$

where

$$
A_{r}(\tau)=\sum_{i=0}^{I-1} W_{I}^{i i} a_{i}(\tau), \tau \in Z\left(T_{1}\right)
$$

which represents a DFT on the $I$ values of the medium rate signals $a_{i}(\tau)$ at each medium-rate instant $\tau=k T+m T_{1}$

\section{Time rearrangement}

The argument of the medium-rate signals $A_{r}(\tau)$ can be written in the form

$$
\tau=k T+m T_{1}=(k M+m) T_{1} \stackrel{\stackrel{1}{=}}{n} n T_{1},
$$

where the integer $n$ uniquely determines both $k$ and $\mathrm{m}$ by the relation

$$
k=\left[\frac{n}{M}\right]=\text { integer division of } n \text { by } M
$$

$$
m=\beta_{M}(n)=\text { remainder of the division of } n \text { by } M .
$$

To introduce the modification in the argument of the filters, we make the following choice

$$
g_{m}\left(t_{0)}=h\left(t_{0}-m T_{1}\right) \alpha_{m}, t \in Z\left(T_{0}\right)\right.
$$

60 
where $h\left(t_{0}\right)$ is the kernel of the interpolating filters in the SCS and , $\alpha_{m}$ is a parameter available for further simplifications. Hence:

$$
\begin{aligned}
u\left(r T_{0}\right)=\sum_{k=-\infty}^{+\infty} \sum_{\substack{m=0 \\
+\infty}}^{M-1} T h\left(r T_{0}-\left(k T+m T_{1}\right)\right) \alpha_{m} W_{N}^{\prime \prime \prime} A_{r}\left(k T+m T_{1}\right) & \\
=\sum_{n=-\infty} T h\left(r T_{0}-n T_{1}\right) \alpha_{m} W_{N}^{m} A_{r}\left(n T_{1}\right) &
\end{aligned}
$$

where $m=\beta_{M}(n)$.

In this relation the $M$ inputs $A_{r}\left(n T_{1}\right)$ are medium-rate signals and the output is a high-rate signal. The latter can be transformed into $M$ medium-rate signals by a $\mathrm{S} / \mathrm{P}$ conversion. In such a way we get an $M$-input $M$-output relation on the common time-domain $Z\left(T_{l}\right)$. To this end let

$$
\begin{aligned}
& r T_{0}=(s I+i) T_{0}=s T_{1}+i T_{0}, s \in \mathbb{Z}, 0 \leq i \leq I-1, \\
& u_{i}\left(s T_{1}\right)=u\left(s T_{1}+i T_{0}\right), h_{i}\left(s T_{1}\right)=\frac{T}{T_{1}} h\left(s T_{1}+i T_{0}\right) .
\end{aligned}
$$

Then:

$$
u_{i}\left(s T_{1}\right)=\sum_{n=-\infty}^{+\infty} T_{1} h_{i}\left(s T_{1}-n T_{1}\right) \alpha_{m} W_{N}^{(s i+i) m} A_{s I+i}\left(n T_{1}\right)
$$

where $(\sec (28 \mathrm{a})) A_{S I+i}=A_{i}, W_{N}^{(s i+i) m}=W_{M}^{s m} W_{N}^{i m}$, and $($ see $(30)) W_{M}^{s m}=W_{M}^{s n}$. Hence

$$
u_{i}\left(s T_{1}\right)=\sum_{n=-\infty}^{+\infty} T_{1} h_{i}\left(s T_{1}-n T_{1}\right) \alpha_{m} W_{N}^{j m} W_{M}^{s n} A_{i}\left(n T_{1}\right)
$$

where $m=\beta_{M}(n)$. 


\section{E Introduction of the MTM}

In the final modification we introduce the following decomposition

$$
W_{M}^{s m}=\gamma(s) \varepsilon(m) \lambda(s-m)
$$

where $\gamma, \varepsilon$, and $\lambda$ are suitable periodic functions (see below), and we choose the parameter $\alpha_{\mathrm{m}}$ as follows

$$
\alpha_{m}=\frac{1}{\varepsilon(m)}
$$

This yields:

$$
u_{i}\left(s T_{1}\right)=\gamma(s) \sum_{n=-\infty}^{+\infty} T_{1} q_{i}\left(s T_{1}-n T_{1}\right)\left[\phi_{i}(n) A_{i}\left(n T_{1}\right)\right]
$$

where

$$
\begin{gathered}
q_{i}\left(r T_{1}\right) \triangleq h_{i}\left(r T_{1}\right) \lambda(r) \\
\phi(n)=W_{N}^{j \beta M^{(n)}} .
\end{gathered}
$$

The interpretation of (37) is given in Fig. 8: the $i$-th signal $A_{i}(\tau)$ is first modulated by the carrier $\phi_{i}\left(F_{1} \tau\right)$, then filtered by the filter $q_{i}(t)$, and finally modulated by the carrier $r\left(F_{1} t\right)$ to produce the signal $u_{i}(t)$.

Considering decomposition (35) considered as a functional equation, in [8] it is shown that any solution for $\gamma$ with $\gamma(0)=1$ uniquely determines both $\varepsilon$ and $\lambda$ by

$$
\varepsilon(r)=\gamma(-r), \quad \lambda(r)=\frac{1}{\gamma(r)} .
$$

The solution is given by the chirp-transform identity 


$$
W_{M}^{s m}=W_{2 M}^{s^{2}} W_{2 M}^{m^{2}} W_{2 M}^{-(s-m)^{2}}
$$

in which

$$
\gamma(r)=W_{2 M}^{\nu^{2}}, \quad \varepsilon(r)=\gamma(r), \quad \lambda(r)=W_{2 M}^{r^{2}}
$$

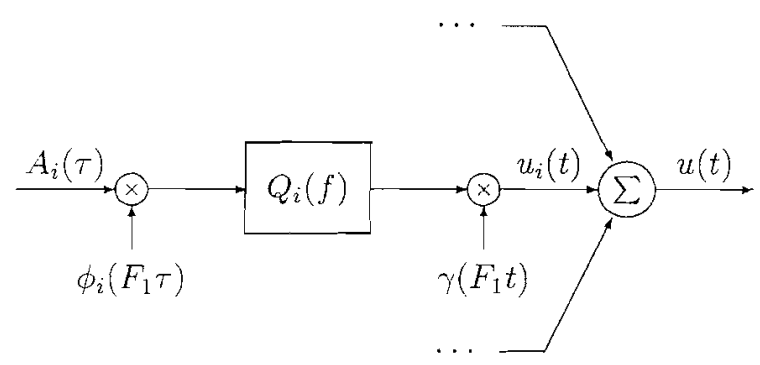

Figure 8 - Interpretation of (37) as a premodulation, a filtering, and a post-modulation.

These functions have period $M$ for $M$ even, and period $2 M$ for $M$ odd. However, in the composite scheme $M$ is confined to even values. ${ }^{6}$ Hence, after $(40)$ the medium-rate carriers $\gamma\left(F_{1} t\right)=\varepsilon\left(F_{1} t\right)$ and $\lambda\left(F_{1} t\right)$ have period $M T_{l}=T$ for any $M \geq 1$.

In Section VI we shall verify that these carriers provide a MTM in the sense outlined in Par. 2-B.

\section{Composite Scheme Formulation}

In the previous section, starting from the SCS overall relationship, we have obtained several partial relationships, which define the composite scheme.

\section{A Summary of relationships}

We now rewrite these partial relationships, after indicating for each one the corresponding components (Fig. 9):

6 A solution with period $M$ for any integer $M$ is

$$
\gamma(r)=W_{2 M}^{r^{2}}(-1)^{M r}
$$


1) group of $M P / S$ conversions, see (25) and Fig. 7

$$
a_{i}\left(t+m T_{1}\right)=s_{i M+m}(t), t \in Z(T), 0 \leq i \leq I-1,0 \leq m \leq M-1
$$

2) $I$-point DFT, see (28a)

$$
A_{r}(\tau)=\sum_{i=0}^{I-1} W_{I}^{\nu i} a_{i}(\tau), t \in Z\left(T_{1}\right)
$$

3) $M T M$ with carrier $\phi_{i}\left(F_{1} t\right)$, see (38b)

4) polyphase filters $Q_{i}(f)$, see (38a)

5) MTM with carrier $\gamma\left(F_{1} t\right)$, see (40). Combination of 3), 4), and 5) yields (see (37), (38), and Fig. 7):

$$
u_{o}(t)=\gamma\left(F_{1} t\right) \sum_{\tau \in Z\left(T_{1}\right)} T_{1} q_{i}(t-\tau)\left[\phi_{i}\left(F_{1} \tau\right) A_{i}(\tau)\right]
$$

6) final P/S conversion, see (33b)

$$
u\left(t+i T_{1}\right)=u_{i}(t), t \in Z\left(T_{1}\right), 0 \leq i \leq I-1
$$

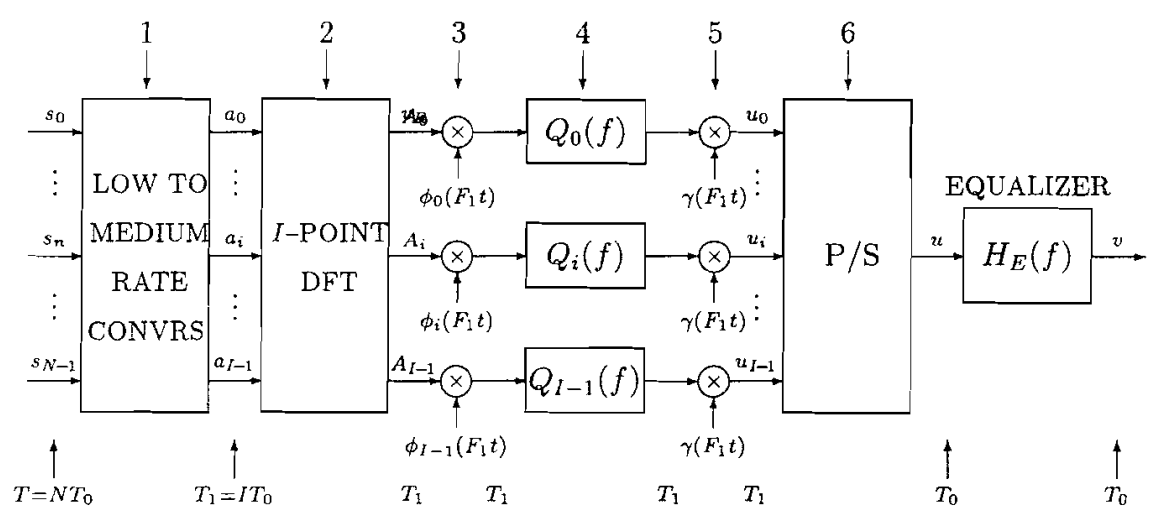

Figure 9 - Components, signals, and time spacing in the "composite" OFDM-SCS scheme. 
7) output equalization, see below

$$
v\left(t_{0}\right)=\left(h_{E} * u\right)\left(t_{0}\right), \quad t_{0} \in Z\left(T_{0}\right)
$$

It is worth noting that components 2) to 5), in which most of the comj utational complexity is confined, works at the medium-rate Fl.

Equalization. The filters $g_{n}\left(t_{0}\right)$, which define the equalizer through condition (21), have been determined in several steps, namely by (27), (31), (36), (39) and (40). Then, its impulse responses are given by

$$
g_{i M+m}\left(t_{0}\right)=\frac{1}{\gamma(m)} h\left(t_{o}-m T_{1}\right)
$$

The corresponding frequency responses are

$$
G_{i M+m}(f)=\frac{1}{\gamma(m)} H(f) e^{-j 2 \pi f m T_{1}}
$$

Hence, from (21), the frequency response of the equalizer is given by

$$
\begin{gathered}
H_{E}(f)=e^{j 2 \pi f \beta_{M}(n) T_{1}} e^{\frac{2 \pi}{2 M} \beta_{M}^{2}(n)} \\
=e^{j 2 \pi f \beta_{M}(n) T_{1}} W_{2 M}^{\beta_{M}^{2} \psi^{(n)}} \quad, f \in \beta_{n} .
\end{gathered}
$$

We now check the extreme cases allowed by the factorizatlon of the number of channels $N=I . M$.

\section{B The case $I=N, M=1$ : scheme via full DFT}

In this case the components 1), 3), 5), and 7) disappear and the scheme becomes composed by

2) an $N$-point $D F T$ at the low-rate $F=l / T$;

4) $N$ low rate filters (polyphase network) with impulse responses

$$
q_{m}(t)=h_{m}(t)=h\left(t-m T_{0}\right), \quad t \in Z(T)
$$


6) a $P / S$ conversion into a high-rate signal.

In conclusion, the scheme is reduced to the one of Fig. la, which gives the OFDM transmitter via an $N$-point DFT and a polyphase network.

\section{The case $M=N, I=1$ : scheme via full MTM}

The components 2), 3), and 6) disappear; hence the scheme becomes:

1) a single $\mathrm{P} / \mathrm{S}$ conversion;

4) a high rate filter (polyphase filter) with impulse response

$$
q(t)=h(t) \lambda\left(F_{0} t\right)=h(t) W_{2 N}^{-\left(F_{0} t\right)^{2}}
$$

5) a high-rate $M T M$ with carrier

$$
\gamma\left(F_{0} t\right)=W_{2 N}^{\left(F_{0}\right)^{2}}
$$

7) output equalization.

In conclusion, the scheme is reduced to the one of Fig. 2b, which gives the OFDM transmitter via a polyphase filter and MTM.

\section{Modification of the composite scheme}

The orders of the final parts 5), 6), and 7) can be changed, as depicted in Fig. 10.

MTM $\gamma$ after the $\mathbf{P} / \mathbf{S}$ conversion. The $I$ identical MTM $\gamma\left(F_{1} t\right)$ work at the medium rate $F_{l}$. It is immediate to check that they can be replaced by a single MTM $\widetilde{\gamma}\left(F_{0} t_{0}\right)$ working at the high rate $F_{0}$ after the $\mathrm{P} / \mathrm{S}$ conversion. The new carrier $\widetilde{\gamma}\left(F_{0} t_{0}\right)$ is given by the $\mathrm{P} / \mathrm{S}$ conversion of the $I$ identical carriers $\gamma\left(F_{1} t\right)$.

Equalization before the $\mathbf{P} / \mathrm{S}$ conversion. The final equalizer can be transferred before the $\mathrm{P} / \mathrm{S}$ conversion at the end of each branch of the composite scheme. This possibility is justified by the fact that $H_{E}(f)$ has period $F_{1}$, whereas a frequency response of a filter working at the rate $F_{0}=M F_{1}$ would have period $F_{0}$. Hence (see Appendix A) the impulse response $h_{E}\left(t_{0}\right), t_{0} \in Z\left(T_{0)}\right.$ is zero except on the multiples of $T_{l}=I T_{0}$ : 
a)

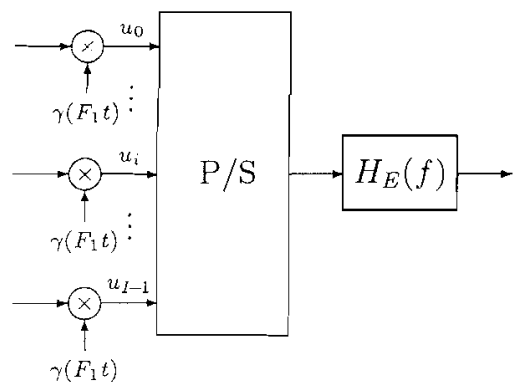

b)

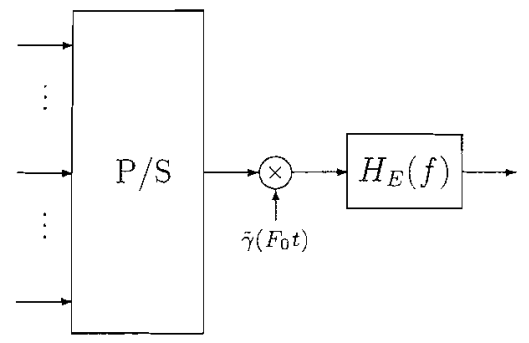

c)

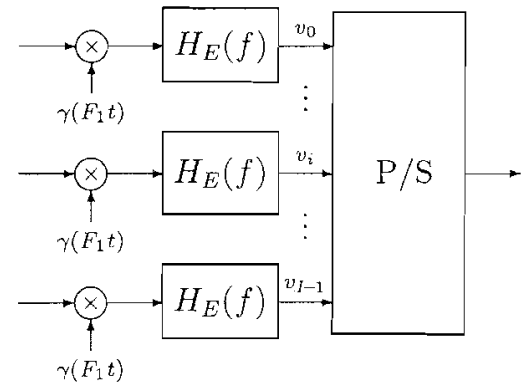

Figure 10 - Possible order change of MTM $\gamma\left(F_{1} t\right)$, P/S conversion, and equalizer in the "composite" OFDM-SCS scheme.a) original configuration, b) with MTM after $\mathrm{P} / \mathrm{S}$, and $\mathrm{c}$ ) with equalization before $\mathrm{P} / \mathrm{S}$.

$$
h_{E}\left(n T_{0}\right)=0, \quad n T_{0} \quad Z\left(T_{1}\right)
$$

Using this property, the input output relationship

$$
\int_{Z\left(T_{0}\right)} d_{\tau_{0}} h_{E}\left(t_{0}-\tau_{0}\right) u\left(\tau_{0}\right)
$$

can be transformed into the form (see Appendix A)

$$
v_{i}(t)=\int_{Z\left(T_{1}\right)} d_{\boldsymbol{\tau}} H_{E}(t-\tau) u_{i}(\tau) \quad, \quad i=0,1, \ldots, I-1
$$

where $v_{i}(t)$ and $u_{i}(\tau)$ are the $\mathrm{S} / \mathrm{P}$ versions of $v\left(t_{0}\right)$ and $u(\tau)$ respectively. The passage from (44) to (45) states that the final high-rate equalizer can be replaced by $I$ identical medium-rate equalizers at the ends of the branches. 


\section{The Memoryless Components}

In this and in the following section we perform the analysis and the synthesis of the components, which essentially are 2), 3), 4), and 5), all working at the medium-rate. In the evaluation of complexity, which strongly depends on the factorization $N=M$. $I$, we take as reference the full DFT scheme. We compare the memoryless part and the parts with memory separately, i.e. 2), 4), and 5) are compared with an $N$-point DFT processor, and 3 ) is compared with an $N$-input $N$-output polyphase network.

\section{A The DFT processor}

This processor must perform an $I$-point complex (inverse) DFT at each medium-rate time $\tau \in Z\left(T_{1}\right)$ according to $(41 \mathrm{c})$. The operation rate when $I$ is a power

of two) is of the order of

$$
R_{D F T}\left(I \log _{2} I\right) F_{1}=\left(\log _{2} I\right) N F
$$

in terms of complex additions and multiplications per second, and a storage requirement of

$$
S_{D F T}=\frac{I}{4}-1 \text { complex coefficients }
$$

Of course, (47) and (48) suggest to choose small values of $I$. Furthermore, for small values of $I$, (47) gives an overestimate of the true complexity. In fact, neglecting additions and trivial multiplications, the DFT processor exhibits no complexity and no storage requirement for $I=2$ and $I=4$, and for $I=8$ it simply requires four multiplications by $W_{8}$ at each medium-rate time, i.e. $4 F_{1}=\left(\frac{1}{2}\right)$ Fo complex multiplications per second (c.m./s). Even, for $I=16$ the DFT processor is considerably simple: $20 \mathrm{c} . \mathrm{m} . / \mathrm{s}$ by $W_{8}$ and $8 \mathrm{c} . \mathrm{m} . / \mathrm{s}$ by $W_{26}$, which lead to the rate of $\left(\frac{7}{4}\right) F_{0} \mathrm{c} . \mathrm{m} . / \mathrm{s}$ in place of 4Fo given by (47). 

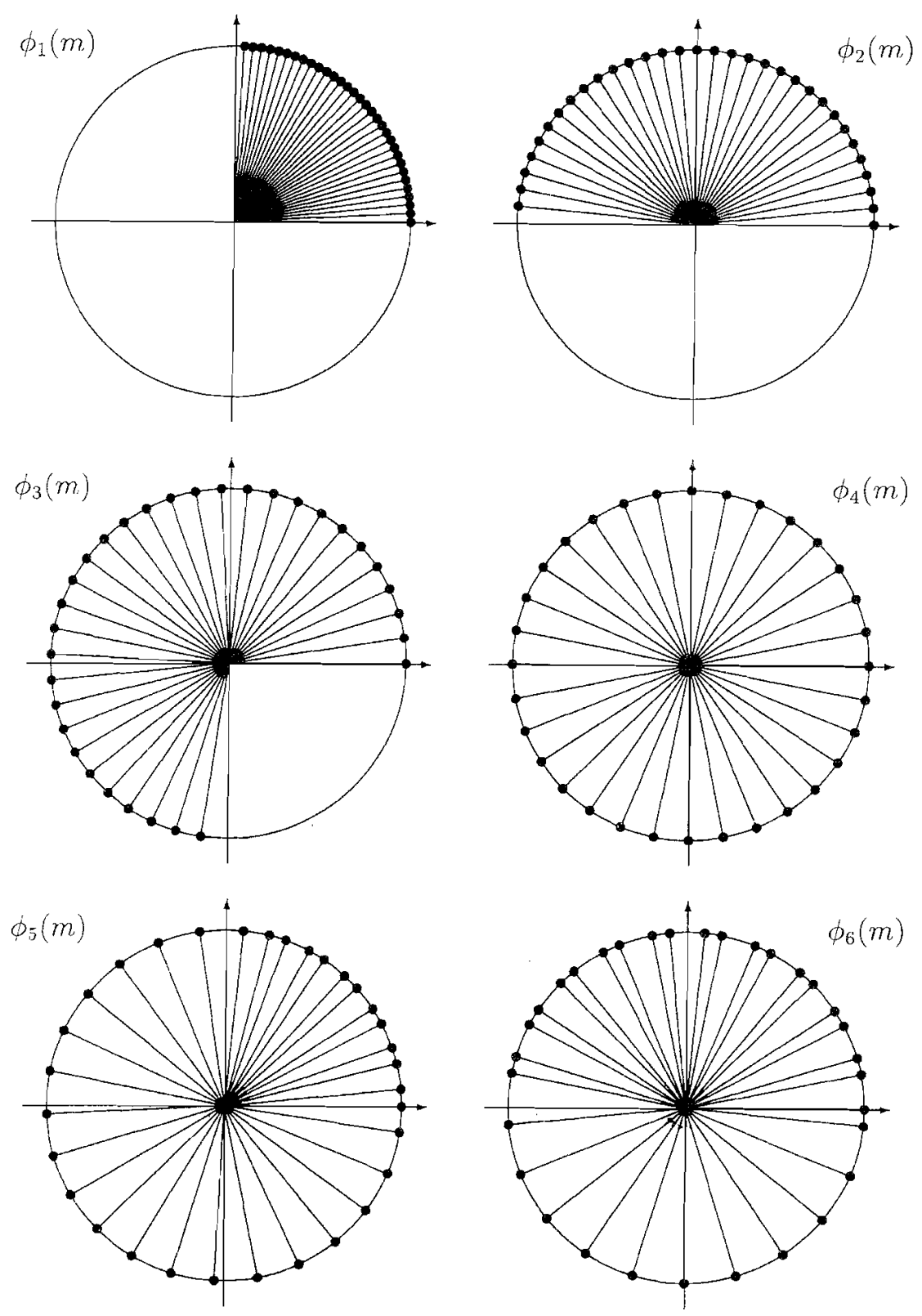

Figure 11 - Constellation of the MTM carriers $\phi_{t}(m)$ in the composite final OFDM scheme N128/M32/14 for some values of $i$. 


\section{B The multitone modulators $\phi_{\mathrm{L}}\left(F_{1} t\right)$}

The carriers of these (I - 1) MTM are defined by (2), namely

$$
\phi_{l}(n)=W_{N}^{i}{ }_{m}(n) \quad, \quad 1 \leq i \leq I-1
$$

and have period $M$ so that $\phi_{1}\left(F_{1} t\right)$ have period $M T_{1}=T$.

The DFT of (49) are easily derived from definition (6a). Considering that, $\beta_{\mathrm{M}}(n)=n$ for $0 \leq n \leq M-1$, they are given by

$$
\Phi_{1}(q)=\frac{1}{M} \frac{1-W_{I}^{i}}{1-W_{N} W_{M}^{-q}} \quad, \quad 0 \leq q \leq M-1
$$

Since $\Phi_{1}(q) \neq 0$ for any $\mathrm{q}$, the above carriers really provide so many MTM according to the considerations of Par. 2 B.

Storage. It is required to store the complex values

$$
W_{N}^{i m}, \quad 1 \leq i \leq M-1, \quad 1 \leq m \leq M-1
$$

and hence, in general, the storage requirement is of

$$
S_{\phi}=(M-1)(I-1) \quad \text { storage values. }
$$

However, neglecting coincidences, trivial multiplications ( $b y \pm 1$ and $\pm j$ ), and conjugation, their number can be considerably reduced. As an example, in N24|M6|I4, where $(M-1)(I-1)=15$, the complete constellations (Fig. 11) can be reproduced by the 5 essential values: $W_{3}, W_{6}, W_{8}, W_{12}, W_{24}$. A similar reduction is found in the other intermediate cases (see Tab. 2). 


\begin{tabular}{|c|c|c|c|c|c|c|c|c|c|c|c|}
\hline \multirow{2}{*}{ M } & \multirow[b]{2}{*}{ I } & \multicolumn{5}{|c|}{$\begin{array}{l}\text { STORAGE } \\
\text { (number of complex coeff.) }\end{array}$} & \multicolumn{5}{|c|}{$\begin{array}{c}\text { OPERATION RATE } \\
\text { (complex multiplies per second) }\end{array}$} \\
\hline & & $S_{D F T}$ & $S_{\varphi}$ & $S_{\gamma}$ & $S_{0}$ & $\frac{S_{0}}{S_{0}^{\prime}}$ & $R_{D F T}$ & $R_{\varphi}$ & $R_{\gamma}$ & $R_{0}$ & $\frac{R_{0}}{R_{0}^{\prime}}$ \\
\hline 1 & 512 & $\begin{array}{l}127 \\
(64)\end{array}$ & - & - & 127 & 1 & $\begin{array}{c}4608 \mathrm{~F} \\
(1538 \mathrm{~F})\end{array}$ & - & - & $\begin{array}{c}4608 \mathrm{~F} \\
(1538 \mathrm{~F})\end{array}$ & 1 \\
\hline 2 & 256 & $\begin{array}{c}63 \\
(56)\end{array}$ & $\begin{array}{l}256 \\
(64)\end{array}$ & $\begin{array}{c}1 \\
(0)\end{array}$ & $\begin{array}{l}319 \\
(64)\end{array}$ & 1 & $\begin{array}{c}4096 \mathrm{~F} \\
(1284 \mathrm{~F})\end{array}$ & $\begin{array}{c}256 \mathrm{~F} \\
(512 \mathrm{~F})\end{array}$ & $\begin{array}{c}256 \mathrm{~F} \\
(0)\end{array}$ & $\begin{array}{c}4607 \mathrm{~F} \\
(1796 \mathrm{~F})\end{array}$ & 1.17 \\
\hline 4 & 128 & $\begin{array}{c}31 \\
(24)\end{array}$ & $\begin{array}{l}381 \\
(64)\end{array}$ & $\begin{array}{c}3 \\
(1)\end{array}$ & $\begin{array}{l}415 \\
(64)\end{array}$ & 1 & $\begin{array}{c}3584 \mathrm{~F} \\
(1032 \mathrm{~F})\end{array}$ & $\begin{array}{c}381 \mathrm{~F} \\
(512 \mathrm{~F})\end{array}$ & $\begin{array}{c}384 \mathrm{~F} \\
(256 \mathrm{~F})\end{array}$ & $\begin{array}{c}4349 F \\
(2800 F)\end{array}$ & 1.17 \\
\hline 8 & 64 & $\begin{array}{l}15 \\
(8)\end{array}$ & $\begin{array}{l}441 \\
(64)\end{array}$ & $\begin{array}{c}7 \\
(1) .\end{array}$ & $\begin{array}{l}463 \\
(64)\end{array}$ & 1 & $\begin{array}{l}3072 \mathrm{~F} \\
(784 \mathrm{~F})\end{array}$ & $\begin{array}{c}441 \mathrm{~F} \\
(512 \mathrm{~F})\end{array}$ & $\begin{array}{c}448 \mathrm{~F} \\
(256 \mathrm{~F})\end{array}$ & $\begin{array}{c}3961 \mathrm{~F} \\
(1552 \mathrm{~F})\end{array}$ & $1+$ \\
\hline 16 & 32 & $\begin{array}{c}7 \\
(4)\end{array}$ & $\begin{array}{l}465 \\
(64)\end{array}$ & $\begin{array}{l}15 \\
(4)\end{array}$ & $\begin{array}{l}487 \\
(64) \\
\end{array}$ & 1 & $\begin{array}{l}2560 \mathrm{~F} \\
(544 \mathrm{~F})\end{array}$ & $\begin{array}{c}465 \mathrm{~F} \\
(512 \mathrm{~F})\end{array}$ & $\begin{array}{c}480 F \\
(512 F)\end{array}$ & $\begin{array}{c}3505 \mathrm{~F} \\
(1568 \mathrm{~F})\end{array}$ & $1+$ \\
\hline 32 & 16 & $\begin{array}{c}3 \\
(2) \\
\end{array}$ & $\begin{array}{l}465 \\
(64) \\
\end{array}$ & $\begin{array}{l}31 \\
(7)\end{array}$ & $\begin{array}{l}499 \\
(64)\end{array}$ & 1 & $\begin{array}{l}2048 \mathrm{~F} \\
(320 \mathrm{~F})\end{array}$ & $\begin{array}{c}465 \mathrm{~F} \\
(512 \mathrm{~F})\end{array}$ & $\begin{array}{c}496 \mathrm{~F} \\
(512 \mathrm{~F})\end{array}$ & $\begin{array}{c}3009 \mathrm{~F} \\
(1344 \mathrm{~F})\end{array}$ & 0.87 \\
\hline 64 & 8 & $\begin{array}{c}1 \\
(1)\end{array}$ & $\begin{array}{l}441 \\
(64)\end{array}$ & $\begin{array}{c}63 \\
(16) \\
\end{array}$ & $\begin{array}{l}505 \\
(64)\end{array}$ & 1 & $\begin{array}{c}1536 \mathrm{~F} \\
(128 \mathrm{~F})\end{array}$ & $\begin{array}{c}441 \mathrm{~F} \\
(512 \mathrm{~F})\end{array}$ & $\begin{array}{c}504 \mathrm{~F} \\
(512 \mathrm{~F})\end{array}$ & $\begin{array}{c}2481 \mathrm{~F} \\
(1152 \mathrm{~F})\end{array}$ & 0.75 \\
\hline 128 & 4 & $\begin{array}{c}0 \\
(0)\end{array}$ & $\begin{array}{l}381 \\
(64) \\
\end{array}$ & $\begin{array}{l}127 \\
(31)\end{array}$ & $\begin{array}{l}508 \\
(64)\end{array}$ & 1 & $\begin{array}{c}1024 \mathrm{~F} \\
(0)\end{array}$ & $\begin{array}{c}381 \mathrm{~F} \\
(512 \mathrm{~F})\end{array}$ & $\begin{array}{c}508 \mathrm{~F} \\
(512 \mathrm{~F})\end{array}$ & $\begin{array}{c}1913 \mathrm{~F} \\
(1024 \mathrm{~F})\end{array}$ & 0.66 \\
\hline 256 & 2 & $\begin{array}{c}0 \\
(0)\end{array}$ & $\begin{array}{l}256 \\
(64)\end{array}$ & $\begin{array}{l}255 \\
(64)\end{array}$ & $\begin{array}{l}510 \\
(64)\end{array}$ & 1 & $\begin{array}{c}512 \mathrm{~F} \\
(0)\end{array}$ & $\begin{array}{c}256 \mathrm{~F} \\
(512 \mathrm{~F})\end{array}$ & $\begin{array}{c}510 \mathrm{~F} \\
(512 \mathrm{~F})\end{array}$ & $\begin{array}{c}1277 \mathrm{~F} \\
(1024 \mathrm{~F})\end{array}$ & 0.66 \\
\hline 512 & 1 & - & - & $\begin{array}{c}511 \\
(127)\end{array}$ & $\begin{array}{c}511 \\
(127)\end{array}$ & 2 & & - & $\begin{array}{c}511 \mathrm{~F} \\
(512 \mathrm{~F})\end{array}$ & $\begin{array}{c}511 \mathrm{~F} \\
(512 \mathrm{~F})\end{array}$ & 0.33 \\
\hline
\end{tabular}

Tab. 2 - Complexity of Memoryless Components $(N=512)$

Multiplies. Since both the input and the output are medium rate signals, each MTM requires $F_{1}=M F \mathrm{c.m} . / \mathrm{s}$, which is reduced to $(M-1) F$ when the multiplications by 1 are neglected. Hence, the operation rate of the whole bank of $I-1$ modulators is

$$
R_{\phi}=(M-1)(I-1) F \mathrm{c.m} . / \mathrm{s} .
$$

This suggests to choose $M$ or $I$ small; in the intermediate case $R_{\phi}$ is of the order of, but less than the high rate $F_{0}$. 
It is worth noticing that $R_{\phi}$ is in any case less than the operation rate of one of the $N$ single-to e modulators appearing in the original scheme of Fig. 3.

\section{The multitone modulators $\gamma\left(F_{1} t\right)$}

The final I identical MTM have the same carrier defined by (3), i.e.

$$
\gamma(n)=W_{2 M}^{n^{2}}(-1)^{M n}=W_{2 M}^{n^{2}} \quad(M \text { even })
$$

which has period $M$ and $\gamma\left(F_{1} t\right)$ has period $M T_{1}=T$.

The DFT is (see [8])

$$
\Gamma(q)=\frac{1}{\sqrt{M}} W_{2 M}^{-q^{2}}
$$

Again $\Gamma(q) \neq 0$ for any $q$ and hence the carrier $\gamma$ provides a MTM.

With reference to the composite scheme of Fig. 9 where these medium-rate MTM operate before the $\mathrm{P} / \mathrm{S}$ conversion, we have seen (Fig. 10) that they can be replaced by a single high-rate MTM $\tilde{\gamma}\left(F_{0} t\right)$ operating after the P/S conversion (Fig. 12). The carrier is just given by the $\mathrm{P} / \mathrm{S}$ conversion of the
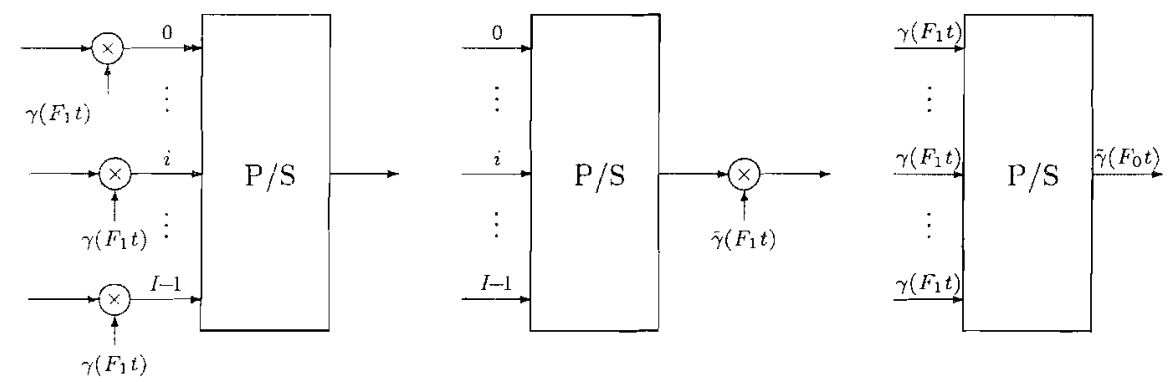

Figure 12 - $a$ ) and b): Substitution of the / identical medium-rate MTM $\gamma\left(F_{1} t\right)$ by a single high rate $\mathrm{MTM} \widetilde{\gamma}\left(F_{0} t\right)$.c) Interpretation of $\widetilde{\gamma}\left(F_{0} t\right)$.

original $I$ identical carriers $\gamma$. This solution, however, does not modify the complexity.

Storage. In general the storage of $\gamma(m), 1 \leq m \leq M-1$, is of 


$$
S_{\gamma_{\gamma}}=M-1 \quad \text { complex values. }
$$

However, as evidenced in [8], $\gamma(m)$ exhibits several coincidences in dependence of the integer $M$. As examples (Fig. 13):

$$
\begin{array}{ll|llllllll}
M=4 & -\frac{m}{\gamma(m)} & 1 & 2 & 3 & & \\
\hdashline & W_{8} & -1 & W_{8} & & & \\
M=8 & m & 1 & 2 & 3 & 4 & 5 & 6 & 7 \\
\hline
\end{array}
$$

\begin{tabular}{c|lrrrrrr}
$m$ & 1 & 2 & 3 & 4 & 5 & 6 & 7 \\
\hline$\gamma(m)$ & $W_{16}$ & $j$ & $W_{8}$ & -1 & $W_{8}$ & $j$ & $W_{8}$
\end{tabular}

In the first case it is needed to store only one value $\left(W_{8}\right)$ and in the second case only two values ( $W_{l 6}$ and $W_{8}$ ). Similar reduction we find for other values of $M$ (see Tab. 2). Note that for any $M<N$ the storage of $\gamma$ modulators is already included in the storage of $\phi_{\mathfrak{l}}$ modulators.

Multiplies. In general, for one $\gamma$ modulator the operation rate is $(M-1) F$ and for the whole bank of $I$ modulators

$$
R_{\gamma}=I(M-1) F \quad \mathrm{c} . \mathrm{m} . / \mathrm{s}
$$

which is less than the high-rate $F_{0}$.

Again, neglecting trivial multiplications, the operation rate turns out to be considerably less than $I(M-1) F$. In the above examples, we find for $M=4$ only $\left(\frac{1}{2}\right) N F$ instead of $\left(\frac{3}{4}\right) N F$, and for $M=8$ only $\left(\frac{3}{8}\right) N F$ instead of $\left(\frac{7}{8}\right) N F$, and similarly for the other cases.

We finally note that, after the MTM, the real part is taken, so that each complex multiply is equivalent to two real multiplications. 

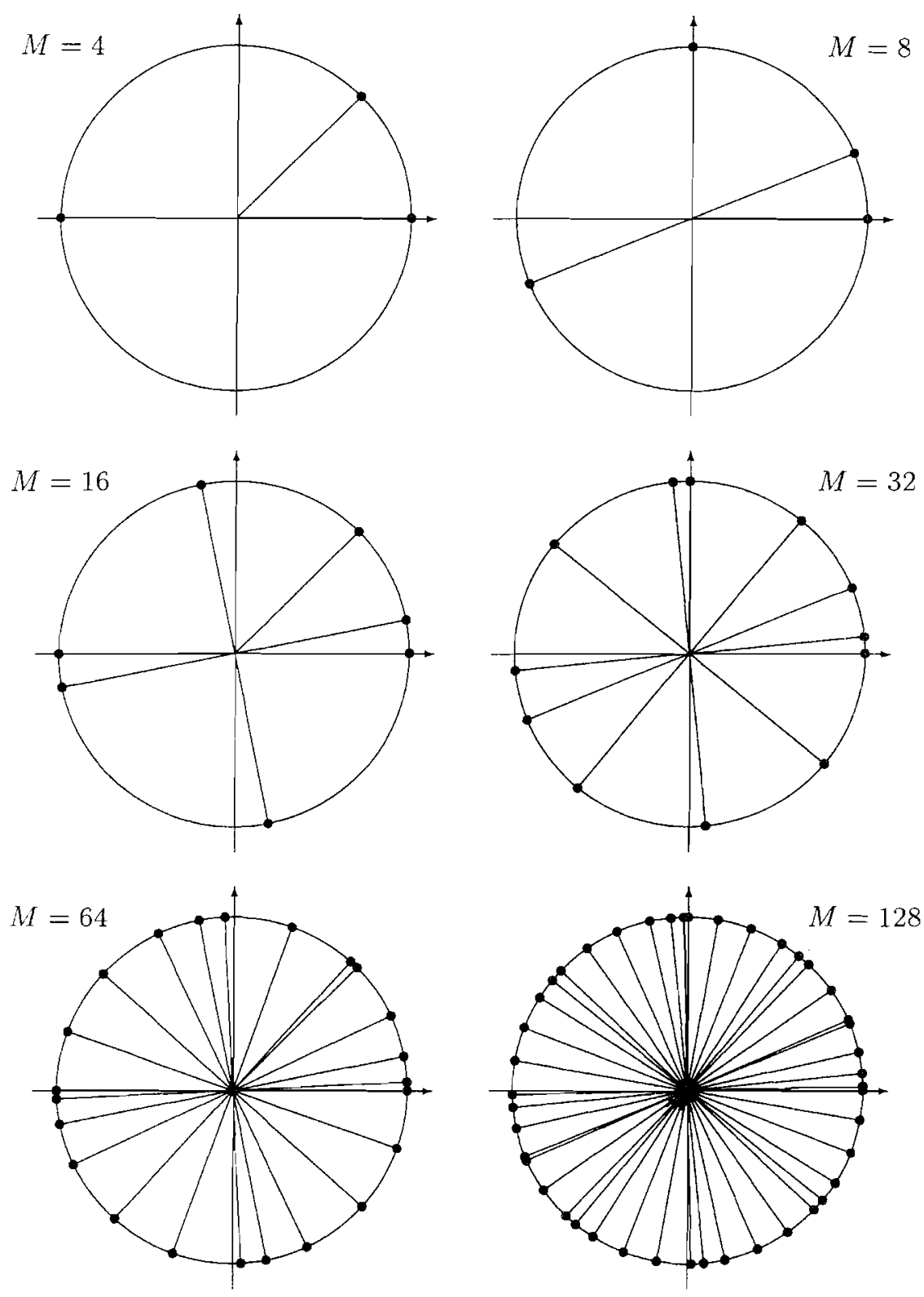

Figure 13 - Constellation of the MTM carriers $\gamma(m)$ for some values of $M$. 


\section{Complexity of the memoryless part}

The storage requirement for the memoryless part of the composite scheme is glven by

$$
S_{0}=S_{D F T}+S_{\phi}+S_{\gamma}=N-\frac{3}{4} I-1
$$

which is compared with the requirements of an $N$-point DFT processor, i.e.

$$
S_{0}^{\prime}=\frac{N}{4}-1
$$

As noted before, for the current values of $\mathrm{N}$, (58) yields a good estimation, whereas (57) yields a rough overestimate for low and intermediate values of I. As an example, in N64|M8|I8 considering the common values in DFT, $\phi_{\mathrm{l}}$ and $\gamma$, we find $S_{0}=8$ values, whereas (58) yields 15 values. Even, in the other cases of interest, we find that for the memoryless part the storage requirements of the composite scheme is of the order of an half with respect to the DFT scheme; moreover the coefficients to store are more simple, as $W_{8}$ and $W_{16}$ instead of $W_{6 t}$.

The operation rate of the memoryless part is given by

$$
R_{0}=R_{D F T}+R_{\phi}+R_{\gamma}=\left(N \log I+2 N-2 I-\frac{N}{I}+1\right) F .
$$

This can be compared with the operation rate of an $N$-point low-rate DFT processor, i.e.

$$
R_{0}^{\prime}=\left(N \log _{2} N\right) F .
$$

We thus find that $R_{0}<R^{\prime}{ }_{0}$ for $I<N$. Again, (59) yields an overestimation for $I<N$, and to obtain the true valuation we have to consider the partial cases of interest. In $N 64|M 8| I 8$ we find (see Tab. 2) $R_{0}=156 F$ and in $N 64|M 16| I 4 R_{0}=92 F$, which must be compared with $R^{\prime}{ }_{0}=384 \mathrm{~F}$. Hence, respectively, $R_{0} / R^{\prime}{ }_{0}=0.40$ and $R_{0} / R^{\prime}{ }_{0}=0.24$.

As expected, the operation rate of the memoryless part of the composite scheme is an increasing function of the integer $I$ and this suggests to choose I as small as possible in connection with the complexity of the polyphase filters. 


\section{The Polyphase Filters}

\section{A Analysis}

The impulse responses of the I polyphase filters (see (33b) and (38a))

$$
q_{i}(t)=h_{i}(t) \lambda\left(F_{1} t\right), \quad t \in Z\left(T_{1}\right)
$$

can be obtained from the impulse response $h(t), t \in Z\left(T_{0}\right)$, of the reference filter with the following operations (Fig. 14): i) a S/P conversion, which yields $h_{i}(t)$, ii) a MTM with carriers $\lambda\left(F_{1} t\right)$.

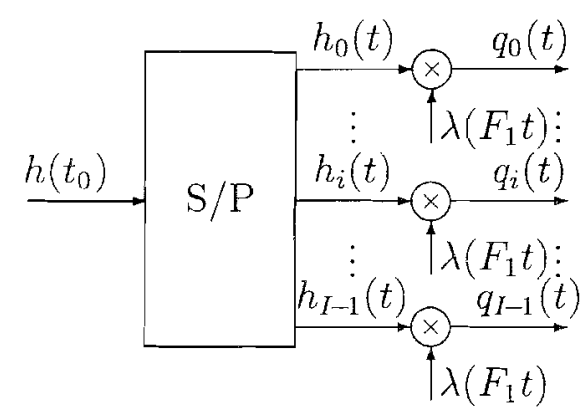

Figure 14 - Interpretation of the impulse responses of the polyphase filters $Q_{i}(f)$

Correspondingly, the transfer function $Q_{i}\left(z^{I}\right)$ can be calculated in terms of $\mathrm{H}(\mathrm{z})$. The $\mathrm{S} / \mathrm{P}$ conversion yields (

$$
H_{i}\left(z^{I}\right)=\frac{1}{I} \sum_{k=0}^{I-1} H\left(z W_{l}^{k}\right) z^{i} W_{I}^{-k i}
$$

and the MTM (see $(8 b))$ :

$$
Q_{i}(s)=\sum_{q-0}^{M-1} \Lambda(q) H_{i}\left(s W_{M}^{-q}\right) \quad, \quad s=z^{I}
$$

76 
where $\Lambda(q)$ is the DFT of $\lambda(n)$, which is given by (see [8]):

$$
\Lambda(q)=\frac{1}{\sqrt{M}} W_{2 M}^{-q^{2}}
$$

Hence

$$
Q_{i}\left(z^{I}\right)=\frac{1}{I} \sum_{q=0}^{M-1} \sum_{k=0}^{I-1} \Lambda(q) H\left(z W_{N}^{-(k M+q)}\right) z^{i} W_{N}^{(k M+q) i}
$$

Letting $n=k M+q$ and considering that $\Lambda(q)=\Lambda(k M+q)$, we also get

$$
Q_{i}\left(z^{I}\right)=\frac{1}{I} \sum_{n=0}^{N-1} \Lambda(n) H\left(z W_{N}^{-n}\right) z^{i} W_{N}^{-n i}
$$

The frequency responses are now obtained by letting $z=\exp \left(j 2 \pi f T_{0}\right)$ and hence $z^{I}=\exp \left(j 2 \pi f T_{1}\right)$, namely

$$
Q_{i}(f)=\frac{1}{I} \sum_{n=0}^{N-1} \Lambda(n) H(f-n F) e^{j 2 \pi f i T_{0} W_{N}^{-n i}}
$$

which has period $F_{1}=M F$.

In the ideal (bandlimited) case the terms in (64) do not overlap and hence

$$
\begin{gathered}
\left|Q_{i}(f)\right|=|H(f-n F)| \\
\arg Q_{i}(f)=\arg \Lambda(0)+\frac{\pi}{m} n^{2}+\pi n M-2 \pi n i+2 \pi f i T_{0}, \\
f \in B_{n}
\end{gathered}
$$

which states that the frequency response $Q_{i}(f)$ has square root raised cosine amplitude with a linear phase in each band $S_{n}$. In particular, the phase at $f=n F$ is 


$$
\arg Q_{i}(n F)=\arg \Lambda(0)+\frac{\pi}{M} n^{2}+\pi n M
$$

and its increment passing from $n F$ to $n F+\frac{1}{2} F$ is

$$
\arg _{i}\left(n F+\frac{1}{2} F\right)-\arg Q_{i}(n F)=\frac{\pi}{N} i
$$

\section{B A preliminary synthesis}

The previous formulas established for the transfer functions $Q_{i}\left(z^{I}\right)$ are not useful for the synthesis of the polyphase filter. Indeed, $Q_{i}\left(z^{l}\right)$ is really a function of $s=z^{I}=\exp \left(j 2 \pi f / F_{1}\right)$, in accordance with the fact that the filters work at the mediumrate $F_{1}$, but (63) expresses $Q_{i}\left(z^{I}\right)$ through functions of $z=\exp \left(j 2 \pi f / F_{0}\right)$. Therefore, if we wish to implement the filters accordingly, we have to use components working at the high-rate $\mathrm{F}_{0}$ with a final decimation. Therefore, we search for other expressions.

a)

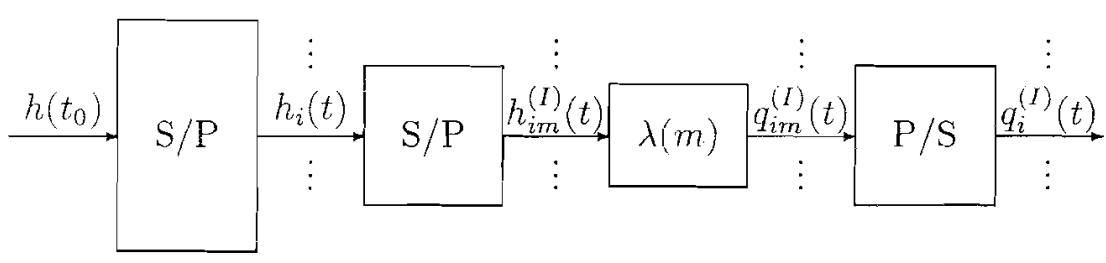

b)

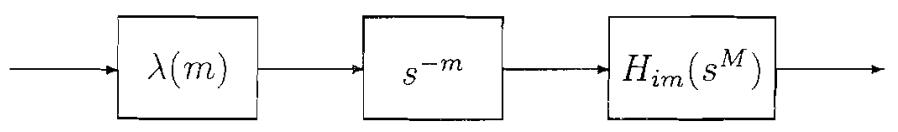

Figure 15 - a) illustration of the relashionships among the components $h_{i}^{(l)}(t)$ and $q_{i}^{(\prime)}(t)$. b) The $m$-th branch of the filter $Q_{l}^{(I)}(f)$.

To reach this goal we rewrite the impulse response (61) in the form

$$
q_{i}^{(I)}\left(n T_{1}\right)=\lambda(n) h_{l}^{(I)}\left(n T_{1}\right)
$$


to remark that $h_{l}^{(I)}$ and $q_{l}^{(I)}$ have been obtained from $h$ and $q$ by a S/P conversion into I components (see Fig. 14). Then, we decompose each $q_{l}^{(l)}$ by a further $\mathrm{S} / \mathrm{P}$ conversion into $M$ components (Fig. 15a) by letting $n T_{1}=(k M+m) T_{1}=k T+m T_{1}$ with $-\infty<k<+\infty$ and $0 \leq m \leq M-1$. Considering the periodicity of $\lambda(n)$, we thus get the following low-rate impulse responses:

$$
q_{i m}^{(N)}(k T)=\lambda(m) h_{i m}^{(N)}(k T)
$$

where now $\lambda(m)$ is independent of the time $k T$, and hence in the zeta domain we get:

$$
Q_{i m}^{(N)}\left(s^{M}\right)=\lambda(m) H_{i m}^{(N)}\left(s^{M}\right)
$$

Since the $M$ signals $q_{i m}^{(N)}, 0 \leq m \leq M-1$, yield $q_{i}$ by a P/S conversion, using (llb) we get

$$
Q_{i}(s)=\sum_{m=0}^{M-1} s^{-m} Q_{i m}^{(N)}\left(s^{M}\right)=\sum_{m=0}^{M-1} s^{-m} \lambda(m) H_{i m}^{(N)}\left(s^{M}\right)
$$

The last represents a first formula for the synthesis of the filter $Q_{i}(s)$ as the parallel of $M$ branches, where the $m$-th branch consists of (Fig. 15b): i) a multiplication by the constant $\lambda(m)$, ii) a delay of $m T_{l}$, and iii) the filters $H_{m}^{(n)}\left(s^{M}\right)$. The total number of filters $H_{i m}^{(N)}\left(s^{M}\right)$ is $I . M=N$, i.e. equal to the number of channels.

To test the computational complexity we remark that the impulse responses $h_{i m}(N)$ obtained from $h$ through two consecutive S/P conversions, first into $I$ components, and then into $M$ components, can be directly obtained by a single $\mathrm{S} / \mathrm{P}$ into $I . M=N$ components $h_{n}^{(N)}$. The relationship is

$$
h_{i m}^{(N)}=h_{i+1}^{(N)} \operatorname{Im}=h_{h}^{(N)} .
$$

On the other hand, we recall that the polyphase network appearing in the full DFT scheme just consists of $N$ filters whose impulse responses are obtained from $h$ by an $\mathrm{S} / \mathrm{P}$ conversion into $N$ components (see (43)). In conclusion, the $N$ filters $H(M)=H(N)$ are just the same that constitute the polyphase network. 
The importance of the above remark is two fold. First, having at the disposal a design of the polyphase network one can easily obtain the design of the bank of $I$ filters of the present scheme. Second, one can compare the complexity between the two schemes; to this end we remark that in the polyphase network the filters work at the low-rate $F$, whereas in the present case they work at the medium-rate $F_{1}=\mathrm{MF}$. In conclision, even if we neglect the multiplies by $\lambda(m)$ (which really have a negligible complexity for small values of $M$ ), the polyphase filters implemented according to (68) have an higher complexity (of the order of $M$ ) than the polyphase network.

This does not mean, however, that the overall complexity in the composite scheme be higher than in the DFT scheme, since the complexity of the memoryless part is considerably less, so as to compensate the higher complexity of the polyphase filters.

\section{Appendix}

\section{A Transferring the equalizer to the medium rate}

The first assertion is stated by (44). The frequency response $H_{E}(f)$ is defined in $R / Z\left(F_{0}\right)$, where as its true periodicity is $Z\left(F_{l}\right)$. Hence $H_{E}(f)$ is equal to deperiodicized version of some $H_{E}(f)$ defined on $R / Z\left(F_{l}\right)$. The relationship between $\hbar(t)$ and $h_{E}\left(t_{0}\right)$ is a time-interpolation $Z\left(T_{1}\right) \Rightarrow Z\left(T_{0}\right)$, namely

$$
\pi\left(n T_{0}\right)= \begin{cases}\ln \left(n T_{0}\right) & n T_{0} \in Z\left(T_{1}\right) \\ 0 & \text { otherwise }\end{cases}
$$

where $I=T_{I} / T_{0}$ is the amplification factor $[10]$.

The second assertion is the passage from (45) to (46). This is achieved by using the Haar integral identity [8]:

$$
\int_{Z\left(T_{0}\right)} d \tau_{0} f\left(\tau_{0}\right)=\sum_{s=0}^{I-1} \int_{Z\left(T_{1}\right)} d \tau f\left(\tau_{0}+s t_{0}\right)
$$

in (45) together with positions:

$$
t_{0}=t+i T_{1}, \quad t \in Z\left(T_{1}\right), \quad i=0,1, \ldots, I-1
$$


We get

$$
v\left(t+i T_{i}\right)=\sum_{s=0}^{I-1} \int_{Z\left(T_{1)}\right.} d \tau h_{E}\left(t+i T_{0}-\tau-s T_{0}\right) u\left(\tau+s T_{0}\right)
$$

Next, considering the ranges of the argument from (71) it follows that $h_{E}\left(t+i T_{0}-\tau--s T_{0}\right)=0, s \neq i$, and hence

$$
v\left(t+i T_{1}\right)=\int_{Z\left(T_{1}\right)} d \tau h_{E}(t-\tau) u\left(\tau+i T_{0}\right)
$$

which is equivalent to (46).

\section{References}

[1] Special Issues on Transmultiplexer, IEEE Trans. on Comm., Vol. COM 30, No. 7, July 1982.

[2] H. Scheuermann, H. Göckler, "A Comprehensive Survey of Digital Tansmultiplexing Methods" IEEE Proceedings, Vol. 69, No 11, November 1981.

[3] B. Hirosaki, "An Orthogonally Multiplexed QAM System Using Discrete Fourier Transform", IEEE Trans. on Communications, vo. COM-29, No 7, July 1981.

[4] B. Hirosaki, S. Hasegawa, A. Sabato, "Advanced Groupband Data Modem Using Orthogonally Multiplexed QAM Technique", IEEE Trans. on Communications, vol. COM-34, pp 587-592, May 1986.

[5] J.A.C. Bingham "Multicarrier Modulation for Data Transmission: An Idea Whose Time Has Come", IEEE Communications Magazine, pp 5-14, May 1990.

[6] N. J. Fliege, "Orthogonal Multiple Carrier Data Tansmission", European Transactions on Telecommunications, vol. 3, No 3, pp 35-44, May-June 1992.

[7] G. Cariolaro, "An OFDM Sheme with half Complexity", GLOBECOM 1994, San Francisco, Nov. Dic. 1994. 
[8] G. Cariolaro, "General Considerations on the Synthesis of OFDM Systems via DFT and MTM", Dept. of Electronics and Informatics, University, University of Padua, IntemalReport October 1992.

[9] G. Cariolaro, "Synthesis of OFDM Systems via Multitone Modulaion", GLOBECOM 1993, Houston, Nov. Dic. 1993.

[10] G. Cariolaro, "La Teoria Unificata dei Segnali”. Torino, UTET, 1991, Ch.6, p.183.

[11] dTTb: digital Terrestrial Broadcasting, European Community Consortium for digital television.

Gianfranco Cariolaro was born in 1936 and graduated in Electrical Engineering at the Universty of Padova (Italy) in 1969. He received the Libera Docenza in Electrical Communications in 1968 and was appointed full professor in 1975. Presently he is professor of Electrical Communications and Signal Theory at the University of Padova. His main researches are in the fields of data transmission, image, digital television and deep space communications. He is author of several books and particularly of "Unified Signal Theory".

Augusto Costanzi was born in Antrodoco, Italy on January 31, 1955. He received the Dr. degree in Physics from University of L'Aquila, Italy. From 1981 to 1993 he was with Telettra, where he was involved in signal processing and communications systems design. Since 1993 he has been with Italtel where he is involved in OFDM modem design. Dr. Costanzi holds five patents and several conference papers relating to communications and signal processing. 